\title{
A High-promising Inquiry-based Lab Experience in Electronics Using Remote Labs for Higher Education Students
}

\author{
Michail Gargalakos ${ }^{1 *}$, Sofoklis Sotiriou ${ }^{2}$ \\ 'Department of Military Science, Hellenic Army Academy, Vari, Greece, ${ }^{2}$ Department of R\&D, Greek-German Education, Pallini, Greece
}

*Corresponding Author: michel@esd.ece.ntua.gr

\section{ABSTRACT}

Technology enhanced learning applications are used more and more in terms of curriculum in primary and secondary education as it has been shown that they have a positive impact to the learning outcome of youngsters. However, for tertiary education this is not the case, since more traditional methods of teaching are still dominant. Despite this fact, there are cases in which e-tools have been proven to be valuable for tertiary education, especially when the shortage of personnel and adequately equipped laboratories can become a serious obstacle for the teaching process. The recent pandemic has proven that technology can offer a unique channel for keep delivering lessons, seminars, and organizing meetings. Innovative interfaces can offer access to remote labs and offer the opportunity to deliver laboratory classes. The impact of the implementation of such e-tools has been carefully investigated using a large number of tertiary students $(n>100)$ and useful conclusions have been drawn, highlighting that the educational value of technology enhanced learning tools is generally underestimated in the field of tertiary education, especially, nowadays, that the pandemic has highlighted the added value of remote laboratories in the teaching process.

KEY WORDS: tertiary education; e-tools; remote laboratories; coronavirus disease-19

\section{INTRODUCTION}

ـ

Technology enhanced learning applications are becoming more and more an everyday practice in primary and secondary education. Multiple e-tools have been developed either in terms of research projects as freeware or as commercial products that can be obtained for a monthly fee giving the chance to millions of students worldwide to engage in science topics. These e-tools have changed the perceptions of students towards natural sciences and have boosted their interest for science domains such as mathematics, physics, astronomy, chemistry, and biology that were until now considered unattractive to the majority of the youngsters (Pyatt and Sims, 2012; Lazonder and Harmsen, 2016; Smith et al., 2020).

A very interesting and innovative category of technology enhanced learning tools are remote and virtual laboratories that are used to enhance typical classroom activities since they provide the opportunity to students to conduct interactive experiments and visualize them through appealing demonstrations (De Jong et al., 2013). Through this process students can be introduced to inquiry-based learning since they formulate their own research questions, conduct the experiments, and finally draw their own conclusions. A European funded project Go-Lab see http://www.go-labproject.eu/ (Dikke et al., 2014) developed online science laboratories (remote and virtual labs) for large-scale use in primary and secondary school education aiming to boost the interest of young people for classroom activities engaging natural sciences. The very promising results from these implementations led to the research question whether these types of tools could be used in tertiary education under the same or a different educational framework.

In the field of tertiary education, the inclusion of technology enhanced learning tools is not widely utilized for a variety of reasons (Dyrberg et al., 2016; Fornsaglio et al., 2019; Kapici et al., 2020). University lessons at the bachelor level tend toward teaching methods which are more traditional and less interactive when compared with secondary or primary education. The image of a crowded lecture theater is very common in tertiary education making the use of e-tools unpractical and ineffective. However, in courses including laboratory experiments, the use of e-tools is not only desirable but also sometimes it may be the only viable solution confronting the lack of personnel and adequate laboratory infrastructure that support the basic educational needs of students (Fornsaglio et al., 2019; Hurtado-Bermúdez and Romero-Abrio, 2020). The recent pandemic has proven that the use of such tools may be a solution that is able to preserve the integrity and the continuity of the teaching process and at the same time reassure the absolute safety of all the participants.

For this paper, we investigated the impact of a large-scale implementation of the Go-Lab integrated environment in a laboratory course of tertiary education. We present the detailed design of the implementation, the way it was carried out in parallel with the actual course, and the results we obtained from a compact questionnaire answered by the students 
after the use of the e-tools. These results demonstrate the added value of the intervention and highlight that technology enhanced learning tools could be a valuable asset for university courses when there are problems of shortage of teaching personnel or of specific laboratory equipment. Moreover, the combination of an actual laboratory exercise that is carried out by the students with the subsequent usage of a similar virtual remote laboratory environment enhances the students learning process and provides them with a better understanding of the basic principles that are being taught to them. In the next two sections, we will discuss the potential of online labs in the educational process and the transformation of the inquiry process in the online environment using the Go-Lab infrastructure.

\section{THE USE OF ONLINE LABORATORIES FOR INQUIRY-LEARNING OVER DISTANCE}

The core activity in an online lab is an investigation (experimentation or exploration) with real or virtual equipment or the possibility of working directly with the results of such a device. In an online lab, investigation material, real or virtual, is manipulated, and the effects of this manipulation are observed to gain insight into the relationship between variables in the conceptual model underlying the online lab (De Jong et al., 2013). There are three types of online labs. In a virtual laboratory, the investigation is performed by the student with virtual equipment. In a remote laboratory, the investigation is performed with physical equipment that is operated at a distance. In a data set, the manipulation has been done by a third party and outcomes of these investigations can be further analyzed and discussed by the students. In our study, the remote lab virtual instrument systems in reality (VISIR) was selected (see http://www.golabz.eu/lab/visir). The VISIR lab provides an environment in which students can construct and test different circuits with a degree of freedom normally associated with a traditional, hands-on electronics laboratory. The advantage of the VISIR system is that the online workbench offers remote equipment that is identical with the laboratory equipment that the students have already used and extensively tested during their laboratory lessons. It should be noted that the remote laboratory was not a virtual laboratory, but an actual one with real equipment that each student had the opportunity to manipulate remotely as if this equipment was available in front of them on the laboratory's workbench.

The topics that can be taught with the use of this online tool are very similar or even identical to the topics that have already been taught in terms of the actual laboratory lesson. This fact significantly diminishes the time that has to be devoted by the teaching personnel for the introduction to the online environment and permits students to be directly introduced to the implementation of the virtual laboratory exercises.

Even though the VISIR tool offered many ready tested exercises and educational scenarios, the fact that these exercises were in Spanish, German, or English language led the teaching team to the decision to create a new educational scenario in the Greek language presenting the passive filter theory and its applications. For the development of this scenario, the authoring platform of the Go-Lab project http:/graasp.eu/ was used. Graasp space is a free open access platform where the users can create their own learning spaces. This platform hosts a wide bouquet of scenarios for teaching, training, or tutoring purposes for a variety of interdisciplinary science topics. These scenarios are enriched with multimedia content from various web resources enabling end users to interact both with the scenario provider and between themselves. For the presentation of the scenario and the explanation of the assignment and before the commencement of the actual implementation, 2 didactic hours were devoted by the teaching staff.

The central pedagogical approach adopted in Go-Lab is inquiry-learning (Rocard et al., 2006). In inquiry-learning students follow a process in which investigations are crucial and important. In inquiry-learning information is not offered directly to students but it is extracted from an interaction with the experiment in the real world or with a model of the experimental device. This investigation process is guided by a hypothesis and requires interpretation of results and the formulation of conclusions. In Go-Lab, the (guided) inquiry approach was used as it has been shown to be more effective than other lab approaches using cookbook procedures or discovery approaches (De Jong et al., 2013). All three types of lab described above provide students with the opportunity to carry out an inquiry process. However, just providing a lab does not suffice for an effective learning process. The scenario consisted of five parts: (a) The orientation phase which described the goals and the prerequisites of the implementation, (b) the conceptualization phase which consisted of the fundamental filter theory that had already been presented in class, check back review questions, and simple solved exercises for practicing, (c) the investigation phase with the student's assignment, (d) the conclusion phase in which the basic conclusions for the whole implementations were summarized, and (e) the discussion phase in which more advanced unsolved exercises and quiz questions were supplied for those who wanted to emphasize more on filters' applications (Figure 1).

The students' assignment was the core of the whole scenario and foresaw the development of a specific first-order low/highpass passive filter with a different cutoff frequency for each student. In Figure 1, a student has built an online a low-pass filter using a predefined pool of resistors and capacitors and at the same time has calculated theoretically the cutoff frequency of the filter to set this as its sinusoidal input frequency. Each student used different resistors and capacitors values resulting in a different cutoff frequency. It should be noted that the breadboard presented in Figure 1 is identical with the breadboard the students used during their actual laboratory course, a fact that permitted to the students to implement the requested filter very quickly. 


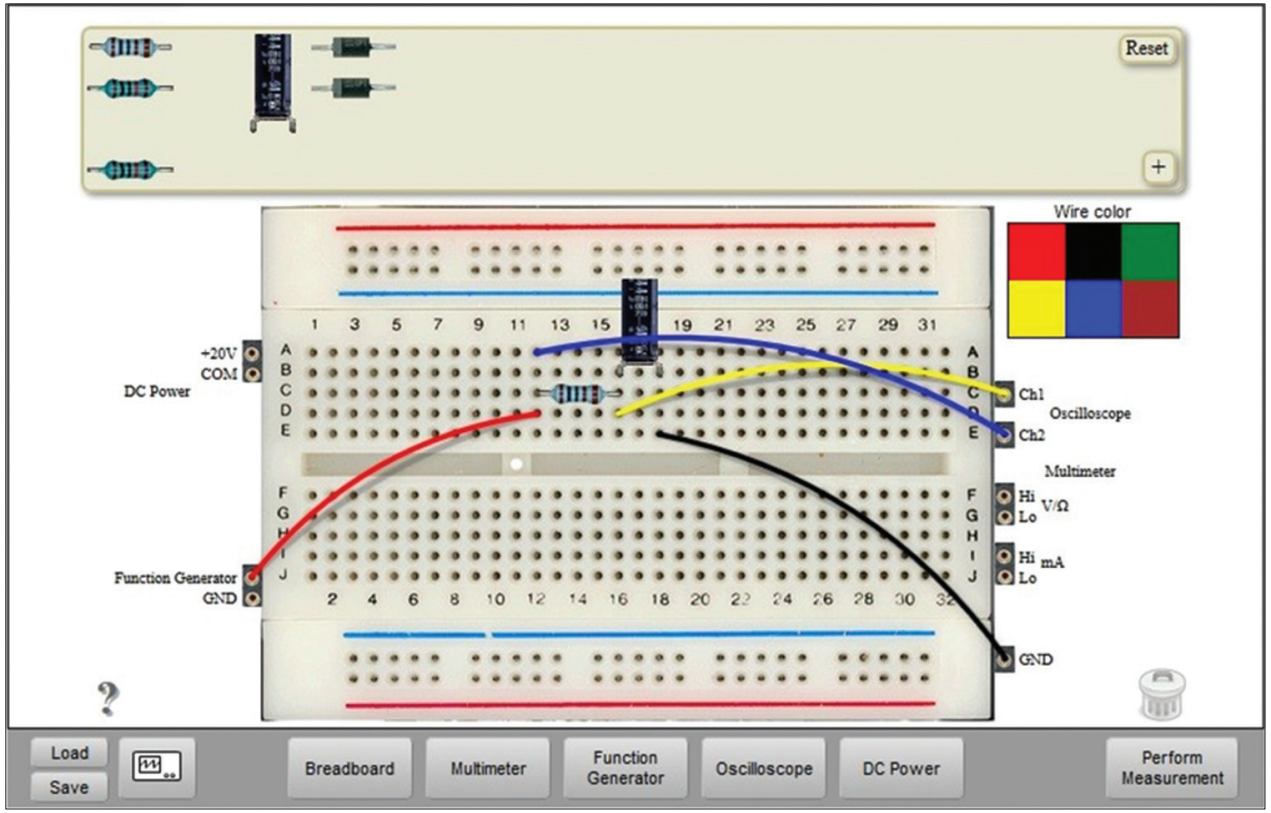

Figure 1: In the framework of the passive filter task students must set up a circuit of a specific first-order low/high-pass passive filter with different cutoff frequency using the virtual instrument systems in reality Remote Lab

The whole process was initiated as the students were voluntarily asked to subscribe to the Go-Lab portal, access the scenario, study it, download the exercise, and then realize it using the remote lab VISIR application. After the development of the filter, each student had to carry out pre-described measurements using different test values and report individually the results using the portal functionalities. In addition, each student was asked to solve the circuit theoretically and compare the theoretical results with those obtained with the use of virtual lab. The aim was to investigate and evaluate possible deviations between theoretical and online results and explore the reasons that had produced these deviations. It should be noted that the same process was used when the students had realized the passive filter during the actual laboratory course and had observed deviations from the theoretically expected results (Figure 2).

For example, as shown in Figure 2, the students were asked to visualize simultaneously both the input and the output of the low-pass/high-pass passive filter to comprehend the $\pm 45^{\circ}$ phase difference imposed by the passive filter between the input and the output sinusoidal signal at the filter's cutoff frequency. Then, they used the remote lab's functionalities to measure this phase difference and they were asked to comment on possible deviations from the theoretically expected phase difference.

\section{DESIGN OF THE IMPLEMENTATION}

The implementation took place for 5 weeks in the Hellenic Army Academy (HAA) during the first semester of the 2019-2020 academic years. The HAA is a Greek tertiary institution where apart from military training the cadets receive a 4-year academic education in topics ranging from history and international relations to mathematics, mechanics, and electronics. The test run was carried out in the $2^{\text {nd }}$ year of HAA ( $3^{\text {rd }}$ semester) in the framework of the electronic laboratory lesson. During the semester, the 230 students were divided into groups of four and had to implement four or five pre-described laboratory exercises. The laboratory lessons took place $2 \mathrm{~h} /$ week and apart from a small theoretical introduction; most of the time was devoted to the implementation of the given exercise with the help of one or two laboratory assistants each time. After the completion of the actual laboratory courses, the predesigned test run took place. The whole test run implementation started at the beginning of December 2019 with a 2-week presentation of all the relevant e-tools by HAA teaching stuff. Then, each student (approximately 155 in total) carried out his own assignment for approximately 5 weeks which lasted from mid December 2019 to January 20, 2020.

The research team informed all participants of the pilot's objectives so that potential aspects that might reasonably be expected to influence their willingness to participate were known and raised in advance. The research team also explained all other aspects of the pilot about which the participants enquired. This was done for participants when asked to join the experiment; consequently, their decision constituted an informed consent. To this end, informed consent and information sheets were presented to students/ participants as well as to their parents or legal representatives for participants aged $<18$ years old, whereas also assent was pursued from the participants themselves. Information sheets were prepared to ensure that the participants' role in the pilot was clear to both the participants themselves and to their parents or legal representatives before the pilot's initiation. Information sheets addressed to students were very brief and used simple language. 


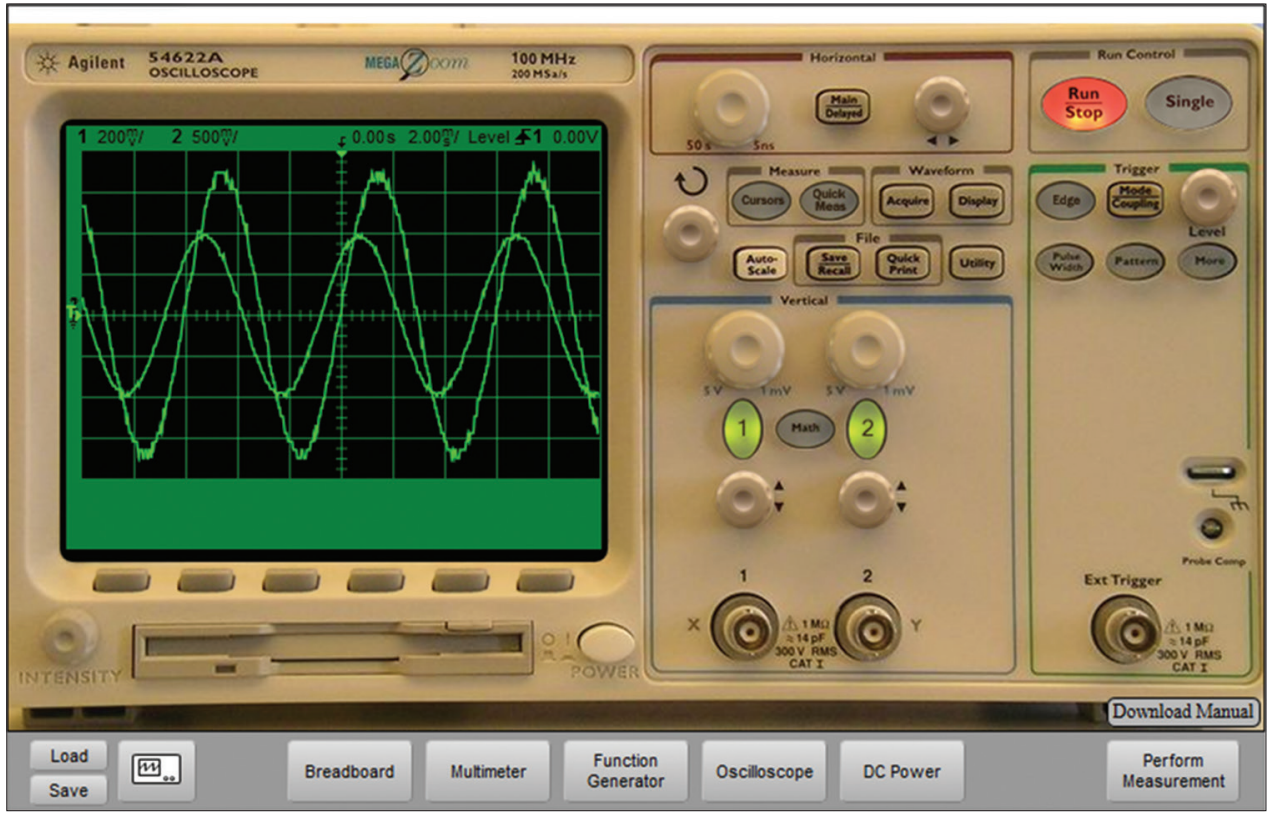

Figure 2: The virtual instrument systems in reality Remote Lab offers the opportunity to visualize the emerging frequencies on an oscilloscope

The topics of the laboratory exercises that were presented and taught during the actual laboratory courses included basic measurements with fundamental electronic equipment such as oscilloscopes, voltage and current meters, multi-meters, power suppliers, and function generators. Furthermore, students created and measured the performance of elementary circuits such as voltage dividers, first-order passive filters, rectifiers, and light-emitting diode. It should be noted that the implementation intentionally took place at the end of the semester, the period when most of the laboratory lessons had been completed. Thus, we could assume that most of the students had already been familiarized with the equipment of the laboratory and the actual measurements.

One of the most demanding topics that were presented to the students during the electronics course both theoretically and practically was the design, construction, and measurement of first-order passive filters. Based on this fact, the research team decided to focus the implementation of the whole test run to the presentation of this specific topic and to try to investigate whether the use of remote labs could have a positive impact on students' understanding of passive filters, given the fact that they had already a previous theoretical and practical knowledge on them.

The whole implementation was carried out by the students on a voluntary basis for the improvement of their final semester grade. Despite the voluntary nature of the implementation, almost $85 \%$ of the students ( 200 out of 230 ) accessed the tool and created an account to try to carry out the exercise and in the end $70 \%$ of them (approximately 155 out of 230 ) managed to submit a final report having completed part or the whole of the assignment. The students had little prior knowledge regarding e-tools for remote laboratories and that created an initial reluctance to undertake the task. In the end the fact that almost $70 \%$ of them decided to submit the assignment, although this was given on a volunteer basis, indicates that the familiarization with the scenario, and the VISIR tool was not as difficult as it initially seemed.

After the completion and the submission of the assignment, all of the participating students were asked to fill in a questionnaire answering a number of questions assessing the use and the impact of the tool, its pedagogical value, and the friendliness of the online environment. The questionnaire consisted of ten questions providing the choice of answering with a number scale ranging from 1 (totally disagree) up to 5 (totally agree) for the posed question. The analyzed sample was 91 students because the questionnaires from the students that had not managed to finish the whole exercise were excluded from the study. The questionnaire was filled in online and the questions were the following:

1. The use of Go-Lab makes the understanding and the learning of physical science easy for me.

2. Using the Go-Lab, I find the lesson of the natural sciences and technology interesting.

3. The use of Go-Lab in the learning of physical science and technology will help me with the everyday practical problems.

4. Using the Go-Lab, the practical exercise in natural sciences is exciting.

5. Using the Go-Lab, the practical exercise in natural sciences is good because I can work together with my collogues.

6. Using the Go-Lab, I like the practical exercise in natural sciences because I can take my own decisions.

7. I would like more practical exercise using the Go-Lab in natural sciences lessons. 
8. We learn natural sciences better when we use the Go-Lab tool.

9. The Go-Lab as an on line learning environment fulfills my needs $\mu$ ov.

10. The use of the Go-Lab environment is easy

Questions 1-8 investigate students' interest and motivation for using this e-tool and its pedagogical impact, while questions 9 and 10 investigate the usage and the user friendliness of the Go-Lab environment. The answers from the questionnaires were correlated with the data gathered from Google analytics showing the timeline of the platform's usage, the type of activity each user had, in which part of the scenario each user focused, and from which place the user's access originated. A special application of the Go-Lab platform included the time spent by each user browsing each part of the scenario. These data were critical because it provided a valuable insight about each user's activity in the Go-Lab portal and helped the research team interpreter more accurately their answers to the questionnaire.

\section{RESULTS}

In the following, we present the results of the students' answers to the selected questions. Table 1 shows the mean values (MV), the Standard Deviations (SD), and the relevant $95 \%$ confidence intervals for questions $1-10$. Figure 3 shows the diagram with the MV and the $95 \%$ confidence intervals for questions

\begin{tabular}{lccc}
\hline \multicolumn{4}{l}{$\begin{array}{l}\text { Table 1: Mean value, Standard deviation, and } \\
\text { confidence intervals of questions } \mathbf{1 - 1 0}\end{array}$} \\
\hline Question no & $\begin{array}{c}\text { Mean value } \\
\text { (MV) }\end{array}$ & $\begin{array}{c}\text { Standard } \\
\text { deviation (SD) }\end{array}$ & $\begin{array}{c}\mathbf{9 5 \%} \text { Confidence } \\
\text { interval }\end{array}$ \\
\hline 1 & 3.81 & 0.87 & 0.177388 \\
2 & 3.98 & 0.80 & 0.163977 \\
3 & 3.81 & 0.84 & 0.172078 \\
4 & 3.80 & 0.80 & 0.164659 \\
5 & 3.85 & 0.84 & 0.172108 \\
6 & 3.81 & 0.91 & 0.185068 \\
7 & 3.76 & 0.94 & 0.19106 \\
8 & 3.84 & 0.87 & 0.178306 \\
9 & 3.76 & 0.75 & 0.153341 \\
10 & 3.67 & 0.72 & 0.146261 \\
\hline
\end{tabular}

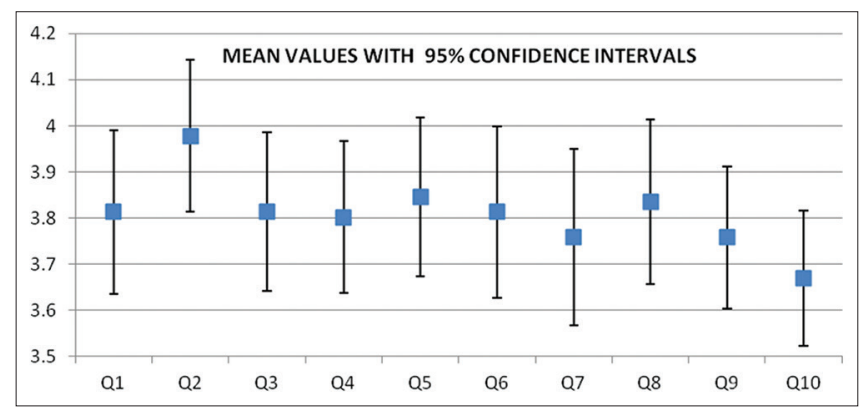

Figure 3: Diagram with mean value and $95 \%$ confidence intervals for questions $1-10$
$1-10$. The answers range from $1=$ totally disagree, $2=$ disagree, $3=$ neither agree nor disagree, $4=$ agree, and $5=$ totally agree. It is remarkable that the MV of the answers to each individual question cluster from 3.70 to 4.00 with SD ranging from 0.7 to 0.95 , while at the same time all the calculated confidence intervals vary within the narrow range of 0.14 to 0.20 . This means that for all questions the answer has a $95 \%$ probability of being within the [3.4-4] interval.

The above results indicate that a large percentage of the participants stated an overall agreement (MV close to 4) to the fact that the Go-Lab tool makes the understanding of the physical sciences easier, more interesting and exciting, helps solving everyday practical problems, enhances team work, and promotes decision making. The students expressed their strong interest to use this environment in other natural science lessons if possible; since they recognized that it enhanced their learning process. As for the user acceptance of the tool itself, despite the fact that not much time was devoted to its presentation, the majority of the participants agreed that the Go-Lab learning environment fulfilled their needs and it was easy and user friendly.

The average time each student spent for browsing the scenario and working with the VISIR tool was a little $<3 \mathrm{~h}$ in total and most of this time was devoted to the investigation phase which consisted of the students' assignment. This calculation is based on the student's time spent on the GRAASP platform in the application. Most of the browsing activity was concentrated 3-4 days before the final date of the assignment submission. Scarce activity was observed during and before Christmas holidays of 2019.

The number of "actions" performed by the users during the implementation was more than 11,000 as they were counted by the data analytics tool. By the term "actions," we refer to users accessing the scenario, downloading material, creating material, printing, logging in, and logging out. About $25 \%$ of these actions were devoted to the investigation phase of the scenario which included the students' assignment, while the rest were dispersed to rest of the scenario phases. In Figure 4, the total actions activity of the users is presented per week for the period between December 2019 and January 2020. The different colors in Figure 4 indicate the time spent for the different phases of the scenario implementation. The blue color that is dominant in the graph is related to the investigation phase, and this is totally understandable, since most of the students spent the majority of their time building and analyzing the filter an action which was associated with the investigation phase. The rest of the colors are associated with the remaining phases of the scenario implementation (orientation, conceptualization, conclusion, and discussion), in which significantly less time was devoted by the participants.

It is evident from this plot that the users' activity was initially limited since students were not so well familiarized with the tool at the beginning of the implementation period and the Christmas holiday period was also an obstacle. This picture is completed reversed during the first 3 weeks of January, when 


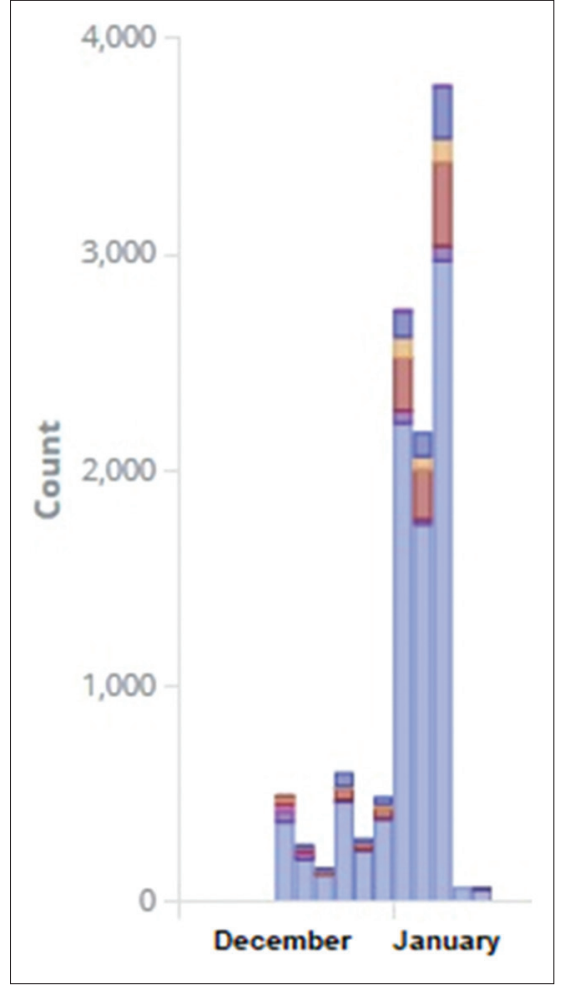

Figure 4: Users activity per week and per scenario phase between December 2019 and January 2020. The blue color refers to the investigation phase; dark blue to the introduction phase; orange to the conceptualization phase; yellow to the conclusion phase; and purple to the discussion phase

the users' activity increased dramatically reaching a peak at the beginning of the $3^{\text {rd }}$ week of January, 1-2 days before the deadline for the submission of the assignment and the final exam of the electronics course. This would indicate that most of the students chose to complete their assignment as close as possible to the date of the course's final exam to take advantage of this opportunity and review their overall knowledge for filters in view of the forearming final written exam.

This tactic was very efficient because after the completion of the written exam and the review of the total scores achieved by the students, we noticed a $20 \%$ improvement to the scores related with filter questions and exercises. More specifically while the mean averages of the electronic course's marks were as expected based on the previous year's record; at the same time, students performed better than the previous exam periods to the part of the exam that was dedicated to the filters. This fact was a clear indication that the whole remote lab implementation improved the total learning outcome of the students for the passive filters. In addition to the previous point, it was noticed that students who had high grades in the final exam, in their majority, were also given high grades for the project.

\section{DISCUSSION}

The introduction of a remote laboratory in the framework of a tertiary course is not a common practice. However, this method has shown its value during our implementation and can solve many practical problems in the future. The lack of personnel for the implementation of actual laboratory courses can be compensated using these tools which have been shown to be effective. The added value is maximized if the students are first introduced to the actual laboratory and then given a hands-on opportunity. Then, they can be asked to use the remote one where experimentations can be performed without the supervision which is necessary during a normal laboratory course.

The impact of the whole intervention to the students' learning outcome was not measured although after the final exam the teaching staff made some very interesting empirical observations. The mean averages of the electronic course's marks were as expected based on the previous year's record; but at the same time, students performed better than previous exam periods to the part of the exam that was dedicated to the filters. In addition to the previous point, it was noticed students who had high grades in the final exam, in their majority were also given high grades for the project.

From a teaching perspective, the experience gained by the teaching staff was valuable since it revealed that this kind of teaching approach if implemented in parallel with the actual laboratory courses can multiply the added value of the hands on experience of the students when they are involved in real measurements with real equipment. In addition, usage of such e-learning tools could also solve many practical problems that usually surface during laboratory courses, such as equipment malfunctions or shortage of trained laboratory personnel. In addition, such e-tools as the Go-Lab permit teachers to assign individual projects to each student and therefore evaluate their knowledge more accurately, while in actual laboratory lessons this in most cases is not possible because students work in groups of 3 or 4 .

The use of this teaching approach supported the teaching staff of the course in addressing an issue raised by previous years' students. The final grade for the electronics' course is the average of a midterm exam, a final exam, and an oral mark that is given to each student for their overall performance throughout the semester, which is basically their performance during the implementation of the actual laboratory exercises. The oral mark has been problematic for the teaching staff due to the number of the students (approximately 230) that must be evaluated and sometimes there are complaints for subjective evaluation.

The possibility that the tool gives to monitor the time that the students spend in each part of the scenario can give to the teachers the opportunity to verify that the students who submit the assignments have actually used the remote laboratory tool and have implemented the scenario. In this sense, each student is forced to work on their own avoiding the temptation of copying other student's assignments. Of course, this verification is not as straight forward as it seems, since other factors have to be also taken in account, but the real time 
monitoring of the students' mobility in the platform is a very good tool that objectifies their performance. That fact leads to the conclusion that if this teaching approach was adopted and introduced officially to the course on a compulsory basis, it could provide alternative and more objective ways for the students' overall oral evaluation.

In addition, the recent pandemic has shown beyond any doubt that these remote labs can provide a unique alternative for carrying on laboratory courses without jeopardizing the learning outcome of the didactical approach. It should be taken into consideration that implementing an actual laboratory course may be the most difficult part of the traditional didactical procedure, since the nature of the laboratory course does not permit the implementation of social distancing and other safety precautions. Therefore, a remote laboratory approach seems as the only feasible solution in view of the necessary actions utilized for preventing the spreading of the coronavirus disease (COVID-19) disease.

Concluding it is evident not only the from analysis of the students' questionnaires but also from the teacher's individual perceptions that the use of such tools in combination with actual similar or even identical laboratory experiments deepened students existing knowledge since they have more available time to experiment individually using the same equipment that they used during the laboratory courses. The crucial difference was that while during the actual laboratory course students have to work in groups of 4 without the possibility of a continuous hands-on experience with the equipment, with the use of the remote laboratory this is made possible, while at the same time the associated COVID-19 spreading risk is eliminated. In addition, working with real equipment can sometimes result in valuable teaching time lost which is spent for the set-up and the calibration of the equipment and the confrontations of equipment malfunctions, something that is totally avoided with the usage of a remote virtual lab.

\section{REFERENCES}

De Jong, T., Linn, M.C., \& Zacharia, Z.C. (2013). Physical and virtual laboratories in science and engineering education. Science, 340(6130), 305-308.

Dikke, D., Tsourlidaki, E., Zervas, P., Cao, Y., Faltin, N., Sotiriou, S., \& Sampson, D. (2014). GOLABZ: Towards a federation of online labs for inquiry-based science education at school. Barcelona, Spain: Proceedings from EDULEARN14, $6^{\text {th }}$ International Conference on Education and New Learning Technologies.

Dyrberg, N.R., Treusch, A.H., \& Wiegand, C. (2016). Virtual laboratories in science education: Students' motivation and experiences in two tertiary biology courses. Journal of Biological Education, 51(4), 358-74.

Fornsaglio, J., Sheffler, Z., Hull, D., \& Bobak, A. (2019). The impact of semester-long authentic research on student experiences. Journal of Biological Education, 1-15. Doi: 10.1080/00219266.2019.1643759.

Hurtado-Bermúdez, S., \& Romero-Abrio, A. (2020). The effects of combining virtual laboratory and advanced technology research laboratory on university students' conceptual understanding of electron microscopy. Interactive Learning Environments, 1-16. Doi: 10.1080/10494820.2020.1821716.

Kapici, H.O., Akcay, H., \& De Jong, T. (2020). How do different laboratory environments influence students' attitudes toward science courses and laboratories? Journal of Research on Technology in Education, 52(4), 534-549.

Lazonder, A.W., \& Harmsen, R. (2016). Meta-analysis of inquiry-based learning: Effects of guidance. Review of Educational Research, 86(3), 681-718.

Pyatt, K., \& Sims, R. (2012). Virtual and physical experimentation in inquiry-based science labs: Attitudes, performance and access. Journal of Science Education and Technology, 21, 133-147.

Rocard, M., Csermely, P., Jorde, D., Lenzen, D., Walberg-Henriksson, H., \& Hemmo, V. (2006). Rocard Report: "Science Education Now: A New Pedagogy for the Future of Europe”. Available from: https://www.eesc. europa.eu/sites/default/files/resources/docs/rapportrocardfinal.pdf.

Smith, E.M., Stein, M.M., Walsh, C., \& Holmes, N.G. (2020). Direct measurement of the impact of teaching experimentation in physics labs. Physical Review X, 10, 011029. 